\title{
A note on the estimation of minimum tracking error portfolios *
}

\author{
PAUlO F. NAibert ${ }^{*}$ \\ Jỗo F. CALDEIRA ${ }^{\dagger}$ \\ AndRÉ A. P. SANTOS ${ }^{\dagger}$
}

\begin{abstract}
Minimum tracking error portfolios are often implemented by portfolio managers in order to track the performance of a benchmark asset in terms of risk and return. This note provides an analytical derivation of the minimum tracking error portfolios of excess returns on a benchmark by relying on the regression-based approach to portfolio weights proposed in Kempf and Memmel (2006). This approach allows estimating the weights of the minimum tracking error portfolios by means of a simple OLS regression.
\end{abstract}

Keywords: Index tracking, portfolio selection, mean-variance analysis

JEL Codes: G11, G12

\section{Introduction}

This note discusses the estimation of the minimum tracking error portfolio (MT). This portfolio policy is among the most used portfolio strategies considered by market practitioners and academics; see, for instance, Chan, Karceski, and Lakonishok (1999), Liu (2009), Brodie, Daubechies, De Mol, Giannone, and Loris (2009) and De Mol (2016). In short, the MT is a passive investment strategy that aims at mimicking the performance of a market benchmark, such as the S\&P 100 or the Ibovespa. When selecting a tracking portfolio, the general goal is to minimize tracking error (TE), which is the difference between index and portfolio returns over time.

The traditional approach to obtain the MT portfolio is based on solving a quadradic optimization problem subjected to user-specified restrictions. We rely

Submitted on 31 May 2019; Reviewed on 26 Dec 2019

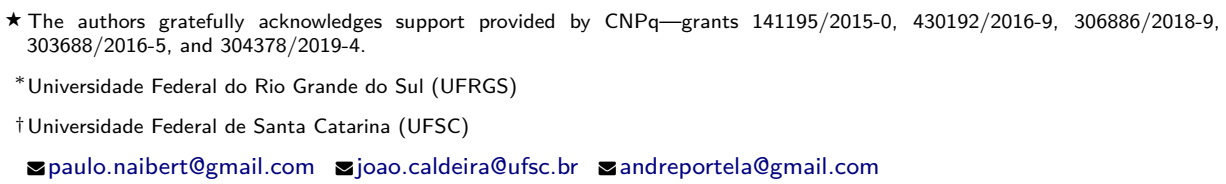


on the work of Kempf and Memmel (2006) to offer an alternative way to obtain the MT portfolio. Kempf and Memmel (2006) obtain the global minimum variance portfolio (GMVP) of risky assets by means of an unconstrained regression. We show that the MT portfolio can be obtained by applying a straightforward manipulation of the regression-based GMVP proposed by Kempf and Memmel (2006). This alternative regression-based approach has at least two advantages with respect to the traditional method. First, it is based on a regression problem that can be easily implemented even in spreadsheets. Second, we can transfer all the distributional results of the OLS approach in order to understand the properties of the conditional distribution of MT portfolio weights.

The rest of this note is structured as follows. In section 2 we review the regression-based approach to the GMVP problem proposed in Kempf and Memmel (2006), propose an extension to the case of the MT problem, and derive the conditional distribution of MT portfolio weights. Section 3 concludes.

\section{Regression approach to the portfolio problem}

This Section describes the regression-based approach to the portfolio problem proposed in Kempf and Memmel (2006). First, we consider the case of the GMVP portfolio. Second, we extend this approach to the case of the MT portfolio. For this purpose, let $N$ be the number of stocks in the capital market. We denote the discrete return of stock $i$ from period $t-1$ to period $t$ by $x_{t, i}$. The vector $\mu$ contains the expected returns, $\mu_{i}$, of the $N$ stocks. The $N \times N$ matrix $\Sigma$ contains the return variances and covariances $\sigma_{i j}$. We assume that the returns are multivariate normally distributed. In addition, we assume they are identically and independently distributed.

\subsection{Global minimum variance portfolios}

In Kempf and Memmel (2006, Proposition 1) the authors put forward a way to obtain the GMVP by means of a OLS regression. Specifically, they rewrite the weights of the GMVP as regression coefficients. For that purpose Kempf and Memmel (2006) set up the problem by choosing the return of stock $N$ to be the dependent variable:

$$
x_{t N}=\alpha+\beta_{1}\left(x_{t N}-x_{t 1}\right)+\cdots+\beta_{N-1}\left(x_{t N}-x_{t, N-1}\right)+\varepsilon_{t}, \quad t=1, \ldots, T .
$$

To solve (1), Kempf and Memmel (2006) define $\boldsymbol{\beta}^{\mathrm{ex}}, \mathbf{w}_{\mathrm{mv}}^{\mathrm{ex}}$, and $\mathbf{x}_{t}^{\mathrm{ex}}$ as $(N-1) \times 1$ vectors. The superscript "ex" indicates that the vector has no entry for asset $N$. 
Morevoer, $\Omega$ is defined as the covariance matrix of regressors of equation (1), i.e. $\Omega=\operatorname{Var}\left(x_{t, N} \mathbf{e}-\mathbf{x}_{t}^{\mathrm{ex}}\right)$ where in this case $\mathbf{e}$ has length $N-1$.

The regression coefficients $\boldsymbol{\beta}^{\text {ex }}$ are the standardized covariances of the regressors and the dependent variable:

$$
\boldsymbol{\beta}^{\mathrm{ex}}=\Omega^{-1} \operatorname{Cov}\left(\mathbf{e} x_{t N}-\mathbf{x}_{t}^{\mathrm{ex}}, x_{t N}\right)
$$

To show that the weights $\mathbf{w}_{\mathrm{mv}}^{\mathrm{ex}}$ of the GMVP correspond the regression coefficient $\boldsymbol{\beta}^{\text {ex }}$, we can compute the weight $w_{\mathrm{mv}, N}$ as $1-\mathbf{e}^{\prime} \mathbf{w}_{\mathrm{mv}}^{\mathrm{ex}}$. For that purpose, it is useful to consider an arbitrary portfolio $p$. Its return is determined by the weight vector $\mathbf{w}_{p}^{\mathrm{ex}}=\left(w_{p, 1}, \ldots, w_{p, N-1}\right)^{\prime}$ and the stock returns $\mathbf{x}_{t}^{\mathrm{ex}}$ and $x_{t, N}$ :

$$
\begin{aligned}
R_{p, t} & =\mathbf{w}_{p}^{\mathrm{ex}} \mathbf{x}_{t}^{\mathrm{ex}}+\left(1-\mathbf{w}_{p}^{\mathrm{ex}} \mathbf{e}\right) x_{t N} \\
& =x_{t N}+\mathbf{w}_{p}^{\mathrm{ex} \prime}\left(\mathbf{x}_{t}^{\mathrm{ex}}-\mathbf{e} x_{t N}\right)
\end{aligned}
$$

The variance of the portfolio excess return of this arbitrary portfolio $p$ is:

$$
\sigma_{p}^{2}=\sigma_{N}^{2}+\mathbf{w}_{p}^{\mathrm{ex}} \Omega \mathbf{w}_{p}^{\mathrm{ex}}-2 \mathbf{w}_{p}^{\mathrm{ex} \prime} \operatorname{Cov}\left(x_{t N} \mathbf{e}-\mathbf{x}_{t}^{\mathrm{ex}}, x_{t N}\right)
$$

To find the weights of the GMVP, we minimize (3) with respect to the portfolio weights $\mathbf{w}_{p}^{\mathrm{ex}}$. This miminization leads to:

$$
\mathbf{w}_{p}^{\mathrm{ex}}=\Omega^{-1} \operatorname{Cov}\left(x_{t N} \mathbf{e}-\mathbf{x}_{t}^{\mathrm{ex}}, x_{t N}\right)
$$

which corresponds to the regression coefficients in (2).

\subsection{Minimum tracking error portfolios}

The MT portfolio policy differs with respect to the GMVP policy in the sense that the former seeks to minimize the variance of the difference between the portfolio returns and the returns of a benchmark asset.

We now discuss how the regression approach to the GMVP problem discussed in subsection 2.1 can be extended to the case of the MT portfolio. First, note that if we replace the returns of asset $N$ at time $t$ by the excess returns of asset $N$ on the benchmark $y$ at time $t$ on equation (1), the solution will be a tracking portfolio and the results of Kempf and Memmel (2006) will hold for this portfolio. Formally, 


$$
\begin{aligned}
& x_{t N}-y_{t}=\alpha+\beta_{1}\left(x_{t N}-x_{t 1}\right)+\cdots \\
& \quad+\beta_{N-1}\left(x_{t N}-x_{t, N-1}\right)+\varepsilon_{t}, \quad t=1, \ldots, T .
\end{aligned}
$$

The regressors in (5) are the same as in equation (1), therefore the covariance matrix of regressors $\Omega$ is defined as before. The left-hand side, however, changes from $x_{t, N}$ to $x_{t, N}-y_{t}$. The vector of beta coefficients of equation (5) is

$$
\boldsymbol{\beta}^{\mathrm{ex}}=\Omega^{-1} \operatorname{Cov}\left(\mathbf{e} x_{t N}-\mathbf{x}_{t}^{\mathrm{ex}}, x_{t N}-y_{t}\right)
$$

Similar as in Kempf and Memmel (2006), we consider an arbitrary portfolio $p$. Its excess return is determined by the weight vector $\mathbf{w}_{p}^{\mathrm{ex}}=\left(w_{p, 1}, \ldots, w_{p, N-1}\right)^{\prime}$, the stock returns $\mathbf{x}_{t}^{\text {ex }}$ and $x_{t, N}$, and the index return $y_{t}$ :

$$
\begin{aligned}
R_{p, t}^{e} & =\mathbf{w}_{p}^{\mathrm{ex}}\left(\mathbf{x}_{t}^{\mathrm{ex}}-\mathbf{e} y_{t}\right)+\left(1-\mathbf{w}_{p}^{\mathrm{ex}} \mathbf{e}\right)\left(x_{t N}-y_{t}\right) \\
& =x_{t N}+\mathbf{w}_{p}^{\mathrm{ex}}\left(\mathbf{x}_{t}^{\mathrm{ex}}-\mathbf{e} x_{t N}\right)-y_{t}
\end{aligned}
$$

The variance of the portfolio return of this arbitrary portfolio $p$ is

$$
\sigma_{p}^{2}=\sigma_{s}^{2}+\mathbf{w}_{p}^{\mathrm{ex}} \Omega \mathbf{w}_{p}^{\mathrm{ex}}-2 \mathbf{w}_{p}^{\mathrm{ex}} \operatorname{Cov}\left(x_{t N} \mathbf{e}-\mathbf{x}_{t}^{\mathrm{ex}}, s_{t}\right)
$$

where $s_{t}=x_{t, N}-y_{t}$. To find the weights of the MT portfolio, we minimize equation (7) with relation to portfolio weights $\mathbf{w}_{p}^{\mathrm{ex}}$. This miminization leads to

$$
\mathbf{w}_{p}^{\mathrm{ex}}=\Omega^{-1} \operatorname{Cov}\left(x_{t N} \mathbf{e}-\mathbf{x}_{t}^{\mathrm{ex}}, x_{t N}-y_{t}\right),
$$

which correspond to the regression coefficients in (6).

\subsubsection{Conditional distribution of portfolio weights}

One interesting aspect of the regression approach to the portfolio problem discussed in section 2 is that we can transfer all the distributional results of the OLS approach in order to understand the properties of the conditional distribution of MT portfolio weights. In Kempf and Memmel (2006, Proposition 3) the authors derive the distribution of the betas in equation (1). To that end, they define the matrix $Z$, which contains regressors $z_{t}=\left(x_{t N}-x_{t, 1}, \ldots, x_{t N}-x_{t N-1}\right)^{\prime}$ of the linear regression (1):

$$
Z:=\left[\begin{array}{cc}
1 & z_{1}^{\prime} \\
\vdots & \vdots \\
1 & z_{T}^{\prime}
\end{array}\right]=\left[\begin{array}{ll}
\mathbf{e} & z
\end{array}\right]
$$


The vector $\bar{z}=T^{-1} \sum_{t=1}^{T} z_{t}$ consists of the arithmetic averages of regressors. In that proposition, the authors state that:

1. The OLS estimates of the portfolio weights $\boldsymbol{\beta}^{\text {ex }}$ are jointly normally distributed:

$$
\hat{\beta}^{\mathrm{ex}} \mid z \sim \mathcal{N}\left(w_{m v}^{\mathrm{ex}} ; \sigma_{m v}^{2}\left(z^{\prime} z-T \bar{z}^{\prime}\right)^{-1}\right)
$$

2. The OLS estimate of the expected return, $\hat{\alpha}$, is normally distributed:

$$
\hat{\alpha} \mid z \sim \mathcal{N}\left(\mu_{m v} ; \sigma_{m v}^{2}\left(1 / T+\bar{z}^{\prime}\left(z^{\prime} z-T \bar{z} \bar{z}^{\prime}\right)^{-1} \bar{z}\right)\right) .
$$

To prove those statements, they observe that the OLS estimator $\hat{B}=$ $\left(\hat{\alpha}, \hat{\beta}_{1}, \ldots, \hat{\beta}_{N-1}\right)=\left(Z^{\prime} Z\right)^{-1} Z^{\prime} x_{N}$, with $x_{N}=\left(x_{1 N}, \ldots, x_{T N}\right)$, is normally distributed:

$$
\hat{B} \mid z \sim \mathcal{N}\left(B ; \sigma_{m v}^{2}\left(Z^{\prime} Z\right)^{-1}\right)
$$

with

$$
Z^{\prime} Z=\left[\begin{array}{cc}
T & T \bar{z}^{\prime} \\
T \bar{z} & z^{\prime} z
\end{array}\right]
$$

The inversion of $Z^{\prime} Z$ yields

$$
\left(Z^{\prime} Z\right)^{-1}=\left[\begin{array}{cc}
1 / T+\bar{z}^{\prime}\left(z^{\prime} z-T \bar{z} \bar{z}^{\prime}\right)^{-1} \bar{z} & \bar{z}^{\prime}\left(z^{\prime} z-T \bar{z} \bar{z}^{\prime}\right) \\
\left(z^{\prime} z-T \bar{z} \bar{z}^{\prime}\right)^{-1} \bar{z} & \left(z^{\prime} z-T \bar{z} \bar{z}^{\prime}\right)^{-1}
\end{array}\right]
$$

which finishes the proof.

In our case, we need to prove that with a change in the regressand, from $x_{N}$ to $s_{N}=x_{N}-y$, so we have:

$$
\hat{B}_{m t}=\left(\hat{\alpha}, \hat{\beta}_{1}, \ldots, \hat{\beta}_{N-1}\right)=\left(Z^{\prime} Z\right)^{-1} Z^{\prime}\left(x_{N}-y\right)=\left(Z^{\prime} Z\right)^{-1} Z^{\prime} s_{N} .
$$

Finally, notice that the same result of Kempf and Memmel (2006, Proposition 3) applies to our case with

$$
\hat{B}_{m t} \mid z \sim \mathcal{N}\left(B_{m t} ; \sigma_{m t}^{2}\left(Z^{\prime} Z\right)^{-1}\right)
$$




\section{Discussion and Final Remarks}

In this note, we provide similar results of Kempf and Memmel (2006, Proposition 1 and 3) to the MT portfolio. Kempf and Memmel (2006) center their focus on the distribution properties of the betas of their regression to test hypothesis about them. Given our equivalence, the same can be done for the MT portfolio. Our result indicate that the challenge concerning portfolio selection for MT can be overcome through the use of a simple regression model.

\section{References}

Brodie, J., Daubechies, I., De Mol, C., Giannone, D., \& Loris, I. (2009). Sparse and stable Markowitz portfolios. Proceedings of the National Academy of Sciences, 106(30), 12267-12272. http://dx.doi.org/10.1073/ pnas.0904287106

Chan, L. K. C., Karceski, J., \& Lakonishok, J. (1999). On portfolio optimization: Forecasting covariances and choosing the risk model. Review of Financial Studies, 12(5), 937-974. http://dx.doi.org/10.1093/rfs/ 12.5.937

De Mol, C. (2016). Sparse Markowitz portfolios. In Financial signal processing and machine learning (pp. 11-22). Wiley-Blackwell. http://dx.doi.org/ 10.1002/9781118745540.ch2

Kempf, A., \& Memmel, C. (2006). Estimating the global minimum variance portfolio. Schmalenbach Business Review, 58(4), 332-348. http:// dx.doi.org/10.1007/BF03396737

Liu, Q. (2009). On portfolio optimization: How and when do we benefit from high-frequency data? Journal of Applied Econometrics, 24(4), 560-582. http://dx.doi.org/10.1002/jae.1062 